\title{
Projective Method For Generic Sensor Fusion Problem $\dagger$
}

\author{
Nageswara S. V. Rao \\ Distributed Intelligent Systems Section \\ Computer Science and Mathematics Division \\ Mailstop 6355, Bldg. 6010 \\ Oak Ridge National Laboratory \\ Oak Ridge, Tennessee 37831-6355 \\ email: raons@ornl.gov
}

\begin{abstract}
"The submitted manuscript has beer suthored by a contractor of the U.S. Government under contract No. DE. AC05-96OR22464. Accordingly, the U.S. Government retains a nonexclu. sive, royalty-free license to publith or reproduce the published form of this contribution, or allow others to do so for U.S. Government purposes."
\end{abstract}

To be presented at the 1999 IEEE/SICE/RSJ International Conference on Multisensor Fusion and Integration for Intelligent Systems, August 15-18, 1999, Taipei, Taiwan.

$\dagger$ Research sponsored in part by the Engineering Research Program of the Office of Basic Energy Sciences, U.S. Department of Energy, under Contract No. DE-AC05-96OR22464 with Lockheed Martin Energy Research Corporation, and the Office of Naval Research under order N00014-96-F-0415. 


\title{
Projective Method For Generic Sensor Fusion Problem
}

\author{
Nageswara S. V. Rao * \\ Center for Engineering Science Advanced Research \\ Computer Science and Mathematics Division \\ Oak Ridge National Laboratory \\ Oak Ridge, TN 378314355
}

\section{A bstract}

In a multiple sensor system, each sensor produces an output which is related to the desired feature according to a certain probability distribution. We propose a fuser that combines the sensor outputs to more accurately predict the desired feature. The fuser utilizes the lower envelope of regression curves of sensors to project the sensor with the least error at each point of the feature space. This fuser is optimal among all projective fusers and also satisfies the isolation property that ensures a performance at least as good as the best sensor. In the case the sensor distributions are not known, we show that a consistent estimator of this fuser can be computed entirely based on a training sample. Compared to linear fusers, the projective fusers provide a complementary performance. We propose two classes of metafusers that utilize both linear and projectives fusers to perform at least as good as the best sensor as well as the best fuser.

\section{Introduction}

In a generic sensor system of $\mathrm{N}$ sensors, the sensor $S_{i}, i=1,2, \ldots, \mathrm{N}$, outputs $Y^{(i)} \in \Re^{d}$ corresponding to input $X \in \Re^{d}$ according to the distribution $P_{Y^{(i)} \mid X}$. The input $\mathrm{X}$ is the quantity that needs to be "estimated" or "measured" by the sensors, such as the presence of a target or a value of the feature vector. The expected error of the sensor $S_{i}$ is given by

$$
I\left(S_{i}\right)=\int C\left(X, Y^{(i)}\right) d P_{Y^{(i)}, X},
$$

where $C: \Re^{d} \times \Re^{d} \mapsto \Re$ is a cost function. $I\left(S_{i}\right)$ is a measure of how good the sensor $S_{i}$ is in "sensing"

\footnotetext{
"This research is sponsored by the Engineering Research Program of the Office of Basic Energy Sciences, U.S. Department of Energy, under Contract No. DE-AC05-960R22464 with Lockheed Martin Energy Research Corp., and the Office of Naval Research under order N00014-96-F-0415.
}

the input feature X. For example, if $S_{i}$ is a target detector or classifier, a choice could be $X \in\{0,1\}$ and $Y^{(i)} \in\{0, I\}$, where $X=1(0)$ corresponds to the presence (absence) of a target. Then

$$
I\left(S_{i}\right)=\int\left[X \oplus Y^{(i)}\right] d P_{Y^{(i)}, X}
$$

corresponds to the probability of misclassification (false alarm or missed detection) of $S_{i}$, where $\oplus$ is the exclusive-OR operation.

This sensor fusion problem is fairly general in that a sensor could be a hardware device, a software module, or a combination. Several other sensor fusion problems can be formulated as special cases. For example, in the door detection problem [8], $S_{i}$ is an ultrasonic or infrared detector, and the objective is to detect doors that are wide enough for a robot to pass through. In the function estimation problems [8], $S_{i}$ is a software module that predicts a function value with certain errors, and the objective is to fuse a number of such modules to improve the prediction accuracy.

There are two types of errors that a sensor can make. The measurement error corresponds to the randomness involved in measuring a particular value of the feature $X$, which is distributed according to $P_{Y^{(i)} \mid X}$. The systematic error at $X$ corresponds to $E\left[C\left(X, Y^{(i)}\right) \mid X\right]$ which must be 0 in the case of a perfect sensor.

We consider a fuser $f: \Re^{N d} \mapsto \Re^{d}$ that combines the outputs of sensors $\mathrm{Y}=\left(Y^{(1)}, Y^{(2)},, Y^{(N)}\right)$ to produce the fused output $\mathrm{f}(\mathrm{Y})$. The expected error of the fuser $f$ is given by

$$
I(f)=\int C(X, f(Y)) d P_{Y, X}
$$

where $\mathrm{Y}=\left(Y^{(1)}, Y^{(2)},, Y^{(N)}\right)$. The objective of fusion is to ensure that $I(f)$ is as small as possible. Note that a fuser must account for both the systematic and measurement errors of the sensors 
in order to achieve a low expected error. In particular, $I(f)$ must be no higher than the error of best sensor, given by $I\left(S_{i^{*}}\right)=\min _{i=1, N .} I\left(S_{i}\right)$. This condition can be achieved by suitably choosing the fusion rule from a function class with the isolation property [6] (see Section 3), which is satisfied by linear combinations and linearized feedforward networks.

In this paper, we propose a projective fuser $f_{L E}$ that is guaranteed to be at least as efficient as the best sensor, and can be much better in certain cases. We also show that this fuser is optimal in the class of projective fusers. This fuser utilizes the lower envelope of the regression curves (with respect to $\mathrm{X}$ ) of sensors by projecting at each $\mathrm{X}$ the sensor with the least error. Similar fusers have been earlier proposed for classifiers [7] and function estimators [9] which are special cases of generic sensors considered in this paper.

Exact computation of $f_{L E}$ requires a complete knowledge of the sensor distributions, which are very difficult to estimate in some engineering and robotic systems. This difficulty is mainly due to the variety and complexity of the present day sensor systems, wherein a single sensor could consist of a number of sensing hardware units and computing modules. As a result, developing accurate error distributions for these systems is extremely challenging, since it could require expertise in a number of disciplines such as device physics, software modules, and statistical estimation. In these systems, however, it is relatively easy to collect "sensor data" by sensing objects with known parameters. We thus consider a formulation wherein the sensor distributions are unknown. We are given an independently and identically distributed (iid) 1-sample

$$
\left(X_{1}, Y_{1}\right),\left(X_{2}, Y_{2}\right), \ldots,\left(X_{l}, Y_{l}\right)
$$

where $Y_{i}=\left(Y_{i}^{(1)}, Y_{i}^{(2)},, Y_{i}^{(N)}\right)$ and $Y_{i}^{(j)}$ is the output of $S_{j}$ in response to input $X_{i}$. In this case, the exact computation of $f_{L E}$ is not possible since it requires a complete knowledge of the sensor distributions. We present a sample-based method to compute a consistent estimator $\hat{f_{L E}}$ of $f_{L E}$ based on regression estimation methods.

In earlier works, the computational and finite sample results have been obtained for the generic fusion problem using fusers based on neural networks, Nadaraya-Watson estimators, and vector spaces (see [8] for an overview). But, very few results that establish the superiority of the fuser over the best sensor based are known for the general case. For the special case of fusing classifiers or concept learners such results are known $[7,10]$. Fur- thermore, most available fusion methods are based on linear fusers $[1,12,3]$. The projective fusers provide a complementary performance and can be used in cases where linear fusers are not effective (see Section 3.1). Furthermore, we propose metafusers that fuse sensors as well as fusers. By suitably utilizing projective and linear fusers, we obtain metafusers that are at least as good as the best sensor as well as the best fuser.

The projective fusers are discussed in Section 2, and are compared to linear, isolation, and metafusers in Sections 3.1, 3.2 and 3.3, respectively. Computation of the projective fusers based on a sample is discussed in Section 4.

\section{Projective Fusers}

Let $\mathrm{Y}=\left(Y^{(1)},, Y^{(N)}\right)$ be the sensor output corresponding to input $\mathrm{X}$. A projective fuser, $f_{P}$, corresponding to a partition $P=\left\{\pi_{1}, \pi_{2}, ., \pi_{k}\right\}$, $k \leq \mathrm{N}$, of input space $\Re^{d}\left(\pi_{i} \subseteq[0,1]^{d}, \bigcup_{i=1}^{k} \pi_{i}=\Re^{d}\right.$, and $\pi_{i} \cap \pi_{j}=\phi$ for $i \neq \mathrm{j}$ ), assigns each block $\pi_{i}$ to a sensor $S_{j}$ such that

$$
f_{P}(Y)=Y^{(j)}
$$

for all $\mathrm{X} \in \pi_{i}$, i.e. the fuser simply transfers the output of the sensor $S_{j}$ for every point in $\pi_{i}$. An optimal projective fuser, denoted by $f_{P^{*}}$, minimizes I(.) over all projective fusers corresponding to all partitions of $\Re^{d}$ and assignments of blocks to sensors $S_{1}, S_{2}, \ldots S_{N}$.

We define the error regression of the sensor $S_{i}$ and fuser $f_{F}$ as $\mathcal{E}\left(X, S_{i}\right)=\int C\left(X, Y^{(i)}\right) d P_{Y \mid X}$ and $\mathcal{E}\left(X, f_{P}\right)=\int C\left(X, f_{P}(Y)\right) d P_{Y \mid X}$, respectively.

Definition 2.1 The projective fuser based on the lower envelope of error regressions of sensors is defined by

$$
f_{L E}(Y)=Y^{\left(i_{L E}(X)\right)}
$$

where

$$
i_{L E}(X)=\underset{i=1,2, \ldots, N}{\arg \min } \mathcal{E}\left(X, S_{i}\right) .
$$

We have $\&\left(\mathrm{X}, f_{L E}\right)=\min _{i=1, \ldots, N} \mathcal{E}\left(X, S_{i}\right)$, or equivalently the error regression of $f_{L E}$ is the lower envelope with respect to $X$ of the set of error regressions of sensors given by $\left\{\mathcal{E}\left(X, S_{1}\right),, \mathcal{E}\left(X, S_{N}\right)\right\}$.

Example 2.1: Consider that $\mathrm{X}$ is uniformly distributed over $[0,1]$, which is measured by two sensors $S_{1}$ and $S_{2}$. Let $C\left(X, Y^{(i)}\right)=\left(\mathrm{X}-Y^{(i)}\right)^{2}$. Consider $Y^{(1)}=\mathrm{X}+|X-1 / 2|+U$ and $Y^{(2)}=$ 
$X+1 /[4(1+|X-1 / 2|)]+U$, where $U$ is an independent random variable with zero mean. Thus, for both sensors the measurement error at any $\mathrm{X}$ is represented by $U$. Note that

$$
\begin{gathered}
E\left[Y^{(1)}-X\right]=|X-1 / 2| \\
E\left[Y^{(2)}-X\right]=1 /[4(1+|X-1 / 2|)] .
\end{gathered}
$$

Thus, $S_{1}$ achieves a low error in the middle of the range $[0,1]$, and $S_{2}$ achieves a low error towards the end point of the range $[0,1]$. The error regressions of the sensors are given by

$$
\begin{gathered}
\mathcal{E}\left(X, S_{1}\right)=(X-1 / 2)^{2}+E\left[U^{2}\right] \\
\mathcal{E}\left(X, S_{2}\right)=1 /\left[16(1+|X-1 / 2|)^{2}\right]+E\left[U^{2}\right] .
\end{gathered}
$$

We have

$$
I\left(S_{1}\right)=0.0833+E\left[U^{2}\right] \text { and } I\left(S_{2}\right)=0.125+E\left[U^{2}\right] \text {, }
$$

which indicates that $S_{1}$ is the better of the two sensors. Now consider the projective fuser $f_{L E}$ specified as follows, which corresponds to the lower envelope of\& $\left(\mathrm{X}, S_{1}\right)$ and $\mathcal{E}\left(X, S_{2}\right)$.

\begin{tabular}{|l|c|}
\hline range for $X$ & sensor to be projected \\
\hline$[0,0.134]$ & $S_{2}$ \\
{$[0.134,0.866]$} & $S_{1}$ \\
{$[0.866,1]$} & $S_{2}$ \\
\hline
\end{tabular}

Then, we have $\mathrm{I}\left(f_{L E}\right)=0.0828+E\left[U^{2}\right]$, which is lower than that of the best sensor.

Example 2.2: We consider a classification example such that $X \in[0,1] \times\{0,1)$ is specified by a function $f_{X}=1_{[1 / 4,3 / 4]}$, where $1_{A}(z)$ is the indicator function (which has a value 1 if and only if $z \in A$ and has value 0 otherwise). The value of $X$ is generated as follows: a random variable $\mathrm{Z}$ is generated uniformly in the interval $[0,1]$ as the first component, and then $f_{X}(Z)$ forms the second component, i.e. $\mathrm{X}=\left(\mathrm{Z}, f_{X}(Z)\right)$. In the context of the detection problem, the second component of $\mathrm{X}$ corresponds to the presence $\left(f_{X}(Z)=1\right)$ or absence $\left(f_{X}(Z)=\right.$ 0 ) of a target, which is represented by a feature $\mathrm{Z}$ taking a value in the interval $[1 / 4,3 / 4]$. Each sensor consists of a device to measure the first component of $\mathrm{X}$ and an algorithm to compute the second component. We consider that $S_{1}$ and $S_{2}$ have ideal devices that measure $\mathrm{Z}$ without an error, but make errors in utilizing the measured features. Consider that $Y^{(1)}=\left(Z, 1_{\left[1 / 4-\epsilon_{1}, 3 / 4\right]}(Z)\right)$ and $Y^{(2)}=$ $\left(Z, 1_{\left[1 / 4,3 / 4-\epsilon_{2}\right]}(Z)\right)$ for some $0<\epsilon_{1}, \epsilon_{2}<1 / 4$ (see Figure 1). In other words, there is no measurement noise in the sensors but just a systematic error due

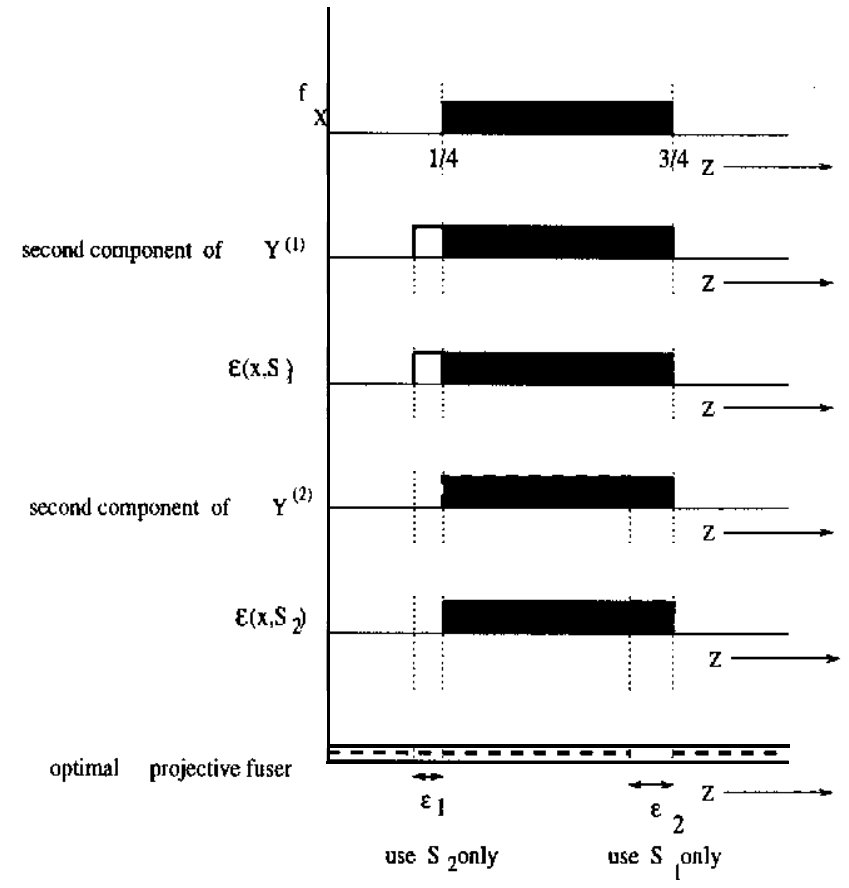

Figure 1: Illustration for Example 2.2.

to how the feature value is utilized; addition of independent measurement noise as in Example 2.1 does not change the basic conclusions of the example. Now consider the quadratic cost function $C\left(X, Y^{(i)}\right)=\left(X-Y^{(i)}\right)^{T}\left(X-Y^{(i)}\right)$. The error regressions are given by $\&\left(X, S_{1}\right)=1_{\left[1 / 4-\epsilon_{1}, 1 / 4\right]}(Z)$ and $\mathcal{E}\left(X, S_{2}\right)=1_{\left\{3 / 4-\epsilon_{2}, 3 / 4\right]}(Z)$, which corresponds to disjoint intervals of $\mathrm{Z}$ as shown in Figure 2. The lower envelope of the two regressions is the zero function hence $I\left(f_{L E}\right)=0$, where as both $I\left(S_{1}\right)$ and $I\left(S_{2}\right)$ are positive. The profile of $f_{L E}$ is shown at the bottom of Figure 1, wherein $S_{1}$ and $S_{2}$ are projected based on the first component of $X$ in the intervals $\left[3 / 4-\epsilon_{2}, 3 / 4\right]$ and $\left[1 / 4-\epsilon_{1}, 1 / 4\right]$, respectively, and in other regions either sensor can be projected.

Theorem 2.1 The projective fuser based on the lower envelope of error regressions as optimal among all projective fusers.

Proof: Let $\mathrm{Y}$ be the sensor output corresponding to input $\mathrm{X}$. For any projective fuser $f_{P}(Y)$, let $i_{P}(X)$ denote the index of the estimator such that $f_{P}(Y)=Y^{\left(i_{P}(X)\right)}$. Then, for $\mathrm{X} \in \Re^{d}$, we have

$$
\begin{aligned}
E\left[C\left(X, f_{P}(X)\right) \mid X\right] & =\mathcal{E}\left(X, f_{P}\right) \\
& =\mathcal{E}\left(X, Y^{\left(i_{P}(X)\right)}\right)
\end{aligned}
$$




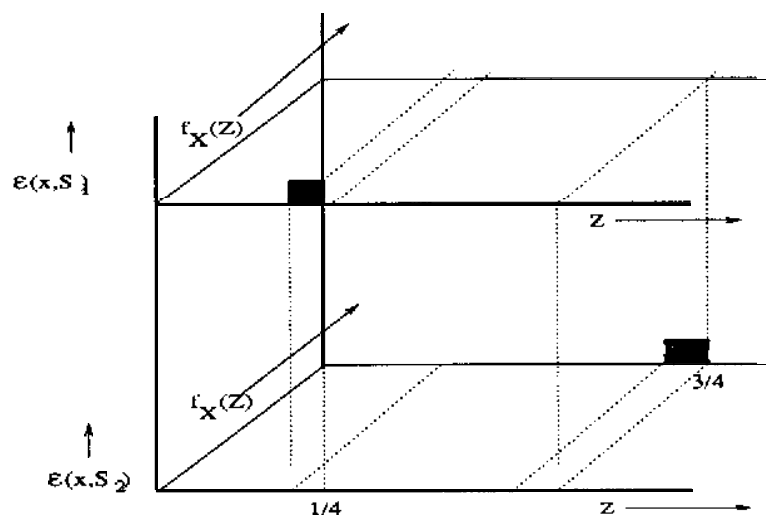

Figure 2: Illustration of error regressions

$$
\begin{aligned}
& \geq \mathcal{E}\left(X, f_{L E}\right) \\
& =E\left[C\left(X-f_{L E}(X)\right) \mid X\right] .
\end{aligned}
$$

By taking the expectations with respect to $P_{X}$ on both sides, we have $I\left(f_{L E}\right) \leq I\left(f_{P}\right)$, and hence $f_{L E}$ is an optimal projective fuser.

\section{Comparison With Other Fusers}

We now compare the performance of the projective fusers with two other types of fusers.

\subsection{Linear Fusers}

The output of linear fuser corresponding to input $\mathrm{X}$ and sensor output $\mathrm{Y}=\left(Y^{(1)},, Y^{(N)}\right)$ is defined as

$$
\mathrm{f}^{\prime}(\mathrm{Y})=\sum_{i=1}^{N} \alpha_{i} Y^{(i)}
$$

where $\alpha_{i}$ is a $d \times d$ matrix. For simplicity, we consider the case $d=1$ such that $\left(\alpha_{1}, \ldots, \alpha_{N}\right) \in \Re^{N}$. An optimal linear combination fuser, denoted by $f_{L^{*}}$, minimizes I(.) over all linear combinations. In terms of relative performance, $f_{L E}$ is better than $f_{L^{*}}$ if the individual sensors perform better in certain localized regions of $\Re^{d}$. On the other hand, if the sensors are equally distributed around certain values in global sense, $f_{L}$ performs better as illustrated follows.

Example 3.1: In the Example 2.2, for $f_{L}=$ $\alpha_{1} Y^{(1)}+\alpha_{2} Y^{(N)}$, we have

$$
I\left(f_{L}\right)=\alpha_{1}^{2} \int_{\left[1 / 4-\epsilon_{1}, 1 / 4\right]} d P_{Z}
$$

$$
\begin{aligned}
& +\left(1-\alpha_{1}-\alpha_{2}\right)^{2} \int_{\left[1 / 4,3 / 4-\epsilon_{2}\right)} d P_{Z} \\
& +\left(1-\alpha_{1}\right)^{2} \int_{\left[3 / 4-\epsilon_{2}, 3 / 4\right]} d P_{Z}
\end{aligned}
$$

which is non-zero no matter what the coefficient are. The error regressions of $S_{1}$ and $S_{2}$ take nonzero values in the intervals $\left[1 / 4-\epsilon_{1}, 1 / 4\right]$ and $[3 / 4-$ $\left.\epsilon_{2}, 3 / 4\right]$ of $Z$, respectively. Since, these intervals are disjoint, there is no possibility of the error of one sensor being canceled by a scaler multiplier of the other.

The argument of Example 3.1 is true in general. If the error regressions of the sensors take non-zero values on disjoint intervals, then any linear fuser will have non-zero error. On the other hand, the disjointness yields $\mathcal{E}\left(X, f_{L E}\right)=0$, for all $\mathrm{X}$, and hence $I\left(f_{L E}\right)=0$. We now present an example where a linear fuser outperforms $f_{L E}$.

Example 3.2: Consider that in Example 2.2, $f_{X}=$ 1 for $Z \in[0,1]$,

$$
\begin{gathered}
Y^{(1)}(X)=(Z, \epsilon Z+1-\epsilon) \\
Y^{(2)}(X)=(Z,-\epsilon Z+1+\epsilon)
\end{gathered}
$$

for $0<\epsilon<1$. The optimal linear fuser is given by $f_{L^{*}}(Y)=1 / 2\left(Y^{\{1)}+Y^{(2)}\right)=\mathbf{1}$, and $I\left(f_{L^{*}}\right)=\mathbf{0}$. At every $X \in[0,1]$, we have

$$
\mathcal{E}\left(X, S_{1}\right)=\mathcal{E}\left(X, S_{2}\right)=\epsilon^{2}(1-Z)^{2}=\mathcal{E}\left(X, f_{L E}\right) .
$$

Thus, $I\left(f_{L E}\right)=\epsilon^{2} \int_{[0,1]}(1-Z)^{2} d P_{Z}>0$, whereas $I\left(f_{L^{*}}\right)=0$.

Note that the addition of independent measurement error as in Example 2.1 by the sensors does not change the basic nature of the conclusion of Examples 3.1 and 3.2. In summary, the performance of the optimal linear and projective fusers are complementary in general.

\subsection{Isolation Fusers}

We now discuss the isolation property of the fuser class that ensures that the fuser is at least as good as the best estimator. A fuser class $\mathcal{G}=\{\mathrm{g}(\mathrm{Y})$ : $\left.\Re^{N d} \mapsto \Re^{d}\right\}$, for $\mathrm{Y}=\left(Y^{(1)},, Y^{(N)}\right)$, has the isolation property with respect to $S_{i}$ if it contains the function $g_{i}(Y)=Y^{(i)}$ for all $i=1,2, ., k$. The isolation property was first proposed in $[10,5]$ for concept and sensor fusion problems. If $\mathcal{G}$ is the set of linear combinations, i. e. $\mathrm{g}(\mathrm{Y})=\alpha_{1} Y^{(1)}++$ 
$\alpha_{k} Y^{\langle k\}}$, for $\alpha_{i} \in \Re$, this property is trivially satisfied for each of $S_{i}, i=1,2, \ldots, k$.

The class of projective fusers satisfies the projection property with respect to $S_{i}$, for $i=1,2, ., k$, since $g_{i}$ corresponds to entire $\Re^{d}$ forming one block assigned to the single sensor $S_{i}$. Consider that $\mathcal{G}=\{\mathrm{g}(\mathrm{Y})\}$ satisfies the. isolation property, then we have for all $i=1,2, \ldots, k$,

$$
\inf _{g \in \mathcal{G}} \int \mathcal{E}(X, g(Y)) d P_{X} \leq \int \mathcal{E}\left(X, Y^{(i)}\right) d P_{X}
$$

which implies $\inf _{g \in \mathcal{G}} \mathrm{I}(\mathrm{g}) \leq \min _{i=1, \ldots . . N} I\left(S_{i}\right)$. Since $I\left(f_{L E}\right) \leq \inf _{g \in \mathcal{G}} \mathrm{I}(\mathrm{g})$ by Theorem 1 , we conclude that $f_{L E}$ performs at least as well as the best sensor.

\subsection{Metafusers}

We now combine linear and projective fusers to realize various configurations that are guaranteed to be at least as good as the best sensor as well as best sensor. By including the optimal linear combination as $S_{N+1}$, we can guarantee that $I\left(f_{L E}\right) \leq$ $I\left(f_{L^{*}}\right)$ by the isolation property of projective fusers (note that $f_{L E}: \Re^{(N+1) d} \mapsto \Re^{d}$ in this case). Since linear combinations also satisfy the isolation property, we in turn have $I\left(f_{L^{*}}\right) \leq \min _{i=1, \ldots, N} I\left(S_{i}\right)$.

The roles of $f_{L^{*}}$ and $f_{L E}$ can be switched - by including $f_{L E}$ as one of the components of $f_{L^{*}}$ to show that

$$
I\left(f_{L^{*}}\right) \leq I\left(f_{L E}\right) \leq \min _{i=1, \ldots, N} I\left(S_{i}\right) .
$$

We close this section by emphasizing that $f_{L E}$ may not be optimal in a larger class of fusers where some function of the sensor output (as opposed to just the output,) can be projected.

Example 3.3: In Example 2.2, consider $f_{X}=$ $1_{[1 / 4,3 / 4]}$,

$$
\begin{gathered}
Y^{(1)}(X)=\left(Z, 1_{\left[1 / 4-\epsilon_{1}, 3 / 4-\epsilon_{1}\right]}(Z)\right) \\
Y^{(2)}(X)=\left(Z, 1_{\left[1 / 4,3 / 4-\epsilon_{2}\right]}(Z)\right)
\end{gathered}
$$

for some $0<\epsilon_{1}, \epsilon_{2}<1 / 8$, and $\epsilon_{1}<\epsilon_{2}$. Thus, we have $\mathcal{E}\left(X, S_{1}\right)=1_{\left[1 / 4-\epsilon_{1}, 1 / 4\right]}(Z)$ and $\mathcal{E}\left(X, S_{2}\right)=1_{\left[3 / 4-\epsilon_{2}, 3 / 4\right]}(Z)$, whose lower envelope is not the zero function. Thus, we have $\mathcal{E}\left(X, f_{L E}\right)=1_{\left[3 / 4-\epsilon_{2}, 3 / 4-\epsilon_{1}\right]}(Z)$ and $I\left(f_{L E}\right)=$ $\left[3 / 4-\epsilon_{2}, 3 / 4-\epsilon_{1}\right]$

$Y^{(1)}$ of $f_{L E}$ to $1-Y^{(1)}$ for $\mathrm{z} \in\left[3 / 4-\epsilon_{2}, 3 / 4-\epsilon_{1}\right]$, one can easily achieve zero error.

\section{Sample-Based Projective Fusers}

The computation of $f_{L E}$ described in the previous section requires a complete knowledge of the distributions of the sensors. To address the case where such knowledge is not available, we compute an estimate $\hat{f}_{L E}$ based on the iid sample. The basic idea is to utilize the regression estimation methods to compute an estimator $\left.\& \hat{(X}, S_{i}\right)$ of $\&\left(X, S_{i}\right)$, and utilize the lower envelope of the estimators in the computation of fuser. Our regression estimation is based on the recent work by Nobel [4] which provides conditions for strong consistency of the regression estimation (weaker forms of consistency are discussed in [11], and stronger conditions are required in [2]). We now outline the basic approach using the cubic partitions with data-dependent offsets for $\mathrm{d}=1$. The same approach can be used for methods based on local fitting with truncated polynomials, K-threshold with variable weights, nearest neighbor clustering, and empirically optimal regression trees (see [4] for details). For a sequence $\left\{h_{l}\right\}$ of positive numbers, consider the partition of $\Re$ given by

$$
\theta_{l}=\left\{\left[(r-1) h_{l}, r h_{l}\right) \mid r \in 2\right) .
$$

Let $\psi_{l}[X]$ denote the unique cell of $\theta_{l}$ that contains $\mathrm{X}$. Then, the estimator of $\mathcal{E}\left(X, S_{i}\right)$ is given by

$$
\hat{\mathcal{E}}\left(X, S_{i}\right)=\frac{\sum_{j=1}^{l} C\left(X_{j}, Y_{j}^{(i)}\right) \mathbf{1}_{\psi_{\mathrm{I}}[X]}\left(X_{j}\right)}{\sum_{j=1}^{n} 1_{\psi_{\mathrm{r}}[X]}\left(X_{j}\right)}
$$

In other words, the estimator simply computes the mean of the error of $S_{i}$ within the cell of $\theta_{l}$ that contains $X$. Consider the conditions: (i) $\mathrm{C}(\mathrm{X}, \mathrm{Y})<\mathrm{K}$ for some $K>0$; (ii) $\lim _{l \rightarrow \infty} h_{l} \rightarrow 0$; and (iii) $n h_{l} \rightarrow \infty$ as $l \rightarrow \infty$. Then, we have $\int\left|\mathcal{E}\left(X, S_{i}\right)-\mathcal{E}\left(X, S_{i}\right)\right|^{2} d P_{X} \rightarrow 0$ with probability 1 [4], regardless of the distributions of sensors.

The fuser $\hat{f}_{L E}$ is defined as in Definition 2.1 with $\hat{\mathcal{E}}\left(X, S_{i}\right)$ used in place of $\mathcal{E}\left(X, S_{i}\right)$. We now show the strong consistency of $\hat{f}_{L E}$ method under the boundedness of $C(.$,$) , namely I\left(\hat{f}_{L E}\right) \rightarrow I\left(f_{L E}\right)$ as $l \rightarrow \infty$ with probability 1 for any sensor distribution. Since $f_{L E}$ is a projective fuser, we have $I\left(f_{L E}\right) \geq I\left(f_{L E}\right) \cdot B Y$ definition ${ }^{\wedge} \mathcal{E}\left(X, f_{L E}\right) \leq$ $\hat{\mathcal{E}}\left(X, f_{L E}\right)$. Then,

$$
\begin{aligned}
& I\left(\hat{f}_{L E}\right)-I\left(f_{L E}\right) \\
& \quad \leq \int\left[\mathcal{E}\left(X, \hat{f}_{L E}\right)-\mathcal{E}\left(X, f_{L E}\right)\right] d P_{X}
\end{aligned}
$$




$$
\begin{aligned}
\leq & \int\left[\mathcal{E}\left(X, \hat{f}_{L E}\right)-\hat{\mathcal{E}}\left(X, \hat{f}_{L E}\right)\right] d P_{X} \\
& +\int\left[\hat{\mathcal{E}}\left(X, \hat{f}_{L E}\right)-\mathcal{E}\left(X, f_{L E}\right)\right] d P_{X} \\
\leq & \int\left|\mathcal{E}\left(X, \hat{f}_{L E}\right)-\hat{\mathcal{E}}\left(X, \hat{f}_{L E}\right)\right| d P_{X} \\
& +\int\left|\hat{\mathcal{E}}\left(X, f_{L E}\right)-\mathcal{E}\left(X, f_{L E}\right)\right| d P_{X} \\
\leq & 2 \sum_{i=1}^{N} \int\left|\mathcal{E}\left(X, S_{i}\right)-\hat{\mathcal{E}}\left(X, S_{i}\right)\right| d P_{X}
\end{aligned}
$$

Since $\int\left|\mathcal{E}\left(X, S_{i}\right)-\hat{\mathcal{E}}\left(X, S_{i}\right)\right| d P_{X} \rightarrow 0$, we have $I\left(\hat{f}_{L E}\right) \rightarrow I\left(\hat{f}_{L E}\right)$ as $l \rightarrow \infty$ with probability 1 . It is interesting to note that this result is valid for any sensor distribution.

\section{Conclusions}

We proposed a fuser that combines the outputs of sensors of a generic sensor system. The fuser utilizes the lower envelope of the regression curves by projecting, at each point of feature space, the sensor with the least error. This fuser is shown to be optimal among all projective fusers, and is also at least as good as the best sensor. In the case the sensor distributions are not known, an estimator of the fuser can be computed entirely based on a training sample. This estimator is shown to be strongly consistent with the optimal projective fuser. We combined the linear and projective fusers to realize metafusers that are guaranteed to be at least as good as the best sensor as well as the best fuser. Open issues in the fuser design are the finite sample performance and computational complexity of the estimator $\hat{f}_{L E}$.

\section{References}

[1] L. Brieman. Stacked regressions. Machine Learning, 24(1):49-64, 1996.

[2] L. Brieman, J. H. Friedman, R. A. Olshen, and C. J. Stone. Classification and Regression Trees. Wadsworth and Brooks, 1984.

[3] M. Mojirsheibani. A consistent combined classification rule. Statistics and Probability Letters, 36:43-47, 1997.

[4] A. Nobel. Histogram regression estimation using datadependent partitions. Annals of Statistics, 24(3):1084-1105, 1996.

[5] N. S. V. Rao. Fusion methods for multiple sensor systems with unknown error densities. Journal of Franklin Institute, 331B(5):509-530, 1994.
[6] N. S. V. Rao. A fusion method that performs better than best sensor. In First International Conference on Multisource-Multisensor Data Fusion. 1998. 19-26.

[7] N. S. V. Rao. To fuse or not to fuse: Fuser versus best classifier. In SPIE Conference on Sensor Fusion: Architectures, Algorithms, and Applications II, pages 25-34. 1998.

[8] N. S. V. Rao. Multiple sensor fusion under unknown distributions. Journal of Franklin Institute, 336(2):285-299, 1999.

[9] N. S. V. Rao. On optimal projective fusers for function estimators. In Second International Conference on Multisource-Multisensor Data Fusion. 1999.

[10] N. S. V. Rao, E. M. Oblow, C. W. Glow, and G. E. Liepins. N-learners problem: Fusion of concepts. IEEE Transactions on Systems, Man and Cybernetics, 24(2):319-327, 1994.

[11] C. J. Stone. Consistent nonparametric regression (with discussion). Annals of Statistics, 5:595-645, 1977.

[12] M. Taniguchi and V. Tresp. Averaging regularized estimators. Neural Computation, 9:11631178, 1997. 